\title{
Programa de charlas empresariales para el desarrollo de capacidades emprendedoras
}

Rosa Elena Cueto Orbe rosacueto.69@gmail.com

Carlos Miguel Aguilar Saldaña Agua0122@hotmail.com

Sandra Ruiz Corea sruiz_correa@hotmail.com

Lady Diana Arévalo Alva Ladydianaarevaloalca85@gmail.com

Luis Alberto Ríos López Rioslopezluisalberto09@gmail.com

Hugo Olivas Salazar holivassalazar@gmail.com

Escuela de post grado RESUMEN

Universidad César Vallejo.

El presente documento de investigación se focaliza esencialmente en conocer la influencia de la aplicación del programa de charlas empresariales en el desarrollo de las capacidades emprendedoras en los alumnos de quinto año de secundaria de la Institución Educativa $N^{\circ} 0381$ “Ángel Custodio García Ramírez” Tarapoto La justificación concuerda con las afirmaciones Formichella (2004), emprendedor es ser capaz de crear algo nuevo o de dar un uso diferente a algo ya existente, y de esa manera generar un impacto en su propia vida y en la de la comunidad en la que habita. El objetivo es determinar la influencia de la aplicación del programa de charlas empresariales en el desarrollo de las capacidades emprendedoras de los alumnos. Se apeló al método el diseño del estudio pre experimental, que ccorresponde al tipo de investigación cuantitativa, del tipo experimental aplicada. Como técnicas de recolección de datos se ha establecido la observación directa y ficha de encuesta. Por último, a través de las charlas empresariales se han desarrollado sus capacidades empresariales, $95 \%$ de confianza.

Palabras clave: emprendimiento; charlas; programa; gestión. 


\title{
Business talks program for the development of entrepreneurial skills
}

\begin{abstract}
This research document focuses essentially on knowing the influence of the application of the business talks program in the development of entrepreneurial skills in fifth-year high school students of the Educational Institution No. 0381 "Ángel Custodio García Ramírez" Tarapoto La justification agrees with the statements Formichella (2004), an entrepreneur is being able to create something new or to give a different use to something already existing, and thus generate an impact on their own life and that of the community in which they live. The objective is to determine the influence of the application of the business talks program in the development of the entrepreneurial capacities of the students. The pre-experimental study design, which corresponds to the type of quantitative research, of the applied experimental type, was used to the method. Direct observation and survey form have been established as data collection techniques. Finally, through the business talks they have developed their business skills, $95 \%$ confidence.
\end{abstract}

Keywords: entrepreneurship; talks; program; management.

Artículo recibido: 10. Junio. 2021 Aceptado para publicación: 16. Julio. 2021 Correspondencia: rosacueto.69@gmail.com Conflictos de Interés: Ninguna que declarar 


\section{INTRODUCCIÓN}

En la actualidad la situación de vida es muy competitiva y globalizada, de tal manera un alumno que termina la secundaria debe salir con una visión positiva de expectativa de vida para realizarse dignamente con una moral en alto, utilizando los dones y recursos de su entorno que se desenvuelve. Las charlas empresariales son una opción muy valiosa que permite al alumno la interactividad y participación activa entre expositor y alumno desarrollando una metodología vivencial con una gran alternativa de vida. En cuanto al desarrollo de capacidades emprendedoras el alumno que termina el quinto año de secundaria no tiene opciones asertivas para realizarse en sí.

En tal motivo el Programa de Charlas Empresariales para el Desarrollo de Capacidades Emprendedoras son cualidades y comportamientos personales claves, necesarios para hacer un(a) emprendedor(a) el cual permite resolver problemas utilizando diferentes estrategias de solución y además ayuda a identificar y aprovechar las oportunidades. Al igual que las personas, las capacidades emprendedoras tampoco son iguales, pues toma en cuenta capacidades que deben desarrollarse como: Autoconfianza, Creatividad, Iniciativa, Perseverancia, Trabajo en equipo y Responsabilidad social, según lo señala Román (2005).

El perfil de formación de los alumnos que egresan del quinto de secundaria es sumamente preocupante debido que el nivel carece de capacidades emprendedores no desarrolladas, el programa de charlas empresariales contribuirá que en cuanto a la capacidad de realización que consiste en el impulso para vencer desafíos, avanzar y crecer al esfuerzo por alcanzar el triunfo, metas y ser útiles a otros. Ver que los estudiantes logren desarrollarse adecuadamente superando los desafíos que se lo evidencian en la práctica de situaciones reales, reforzado con la capacidad de planificación pensando antes de actuar, identificando metas concretas o resultados que se desea alcanzar y diseñar planes consistentes para desempeñarse de manera que se aproveche al máximo las oportunidades anhelando disminuir las amenazas del entorno y finalizando en la capacidad de socialización en que el alumno genera estados de ánimo positivo en las personas de su entorno que se traduzcan en compromisos de apoyo para el desarrollo de sus proyectos, en conclusión del alumno emprendedor. 


\section{Programa de Charlas Empresariales}

La Corporación Financiera de Desarrollo (COFIDE) en su Programa de Capacitación Empresarial - Tabla de Negocios MyPE. Este programa ha sido creado en base a la trayectoria que tiene COFIDE trabajando con los jóvenes emprendedores peruanos. Todo como parte del ecosistema emprendedor al que se quiere llegar. COFIDE ha diseñado, habrá charlas, conferencias, asesorías personalizadas en negocios y más, todo orientado al crecimiento de micro y pequeñas empresas del país.

El programa está compuesto por 20 charlas, las cuales se dividen en los siguientes módulos, las mismas que tienen mayor demanda en los microempresarios: Legal, Contable, Marketing, Administración y Finanzas. Una vez que los asistentes hayan cursado un mínimo de 24 de las 30 horas impartidas, en este caso se tomó este modelo para ser impartido a los estudiantes de la institución educativa.

El programa de capacitación está compuesto por 4 módulos que consisten en los siguientes temas: Legal / Contable

\section{Formalización de Empresas.}

Ley $N^{o}$ 28015, Ley De Promoción y Formalización De La Micro y Pequeña Empresa, menciona en el Artículo $1^{\circ}$ : "La presente ley tiene por objeto la promoción de la competitividad, formalización y desarrollo de las micro y pequeñas empresas para incrementar el empleo sostenible, su productividad y rentabilidad, su contribución al Producto Bruto Interno, la ampliación del mercado interno y las exportaciones, y su contribución a la recaudación tributaria."

Aspectos Contables. Consiste en registrar información, relacionada con el manejo de la empresa, mediante el cual se puede consultar mediante instrumentos tales como libros y registros contables, en un determinado momento de cuál es la situación económica, laboral y productiva de la empresa.

Aspectos Tributarios. Determinar el mejor Régimen tributario, para la empresa, obteniendo un mejor beneficio para la rentabilidad del negocio. Formular presupuestos razonables, que aumenten la seguridad de la MyPE y sobre todo una buena gestión en la generación de fondos. Esto te hará sujeto de crédito y un buen referente para hacer negocios con otras empresas.

Tributación para MyPE I. Régimen tributario de las de las MyPE. Artículo $\mathrm{N}^{\circ} 42^{\circ}$.El Régimen Tributario facilita la tributación de las MyPE y permite que un mayor 
número de contribuyentes de las MyPE se incorpore a la formalidad. El Estado promueve campañas de difusión sobre el régimen tributario, en especial el de aplicación a las MYPE con los sectores involucrados. La SUNAT adopta las medidas técnicas, normativas, necesarias para fortalecer y cumplir su rol de entidad administradora, recaudadora y fiscalizadora de los tributos de las recaudadora y fiscalizadora de los tributos de las MYPE.

Tributación para MyPE II. Tipos de régimen tributario: Régimen general del impuesto a la renta, Régimen especial del impuesto a la renta, Régimen único Simplificado.

\section{Administración}

Gestión de calidad. Se entiende como una estructura operacional de trabajo bien documentado e integrado de los procesos administrativos de las MyPES.

\section{Ventajas que ofrecen los sistemas de calidad.}

Fortalece la Imagen de la empresa, mediante la satisfacción del cliente creando una relación cliente proveedor. Asegura la calidad en las relaciones comerciales. Facilita la exportación de los productos, mediante los estándares de calidad. Mejora la calidad del Producto o servicio. Bajan los costos y suben los ingresos.

Gestión de Competitividad. Se define como la capacidad de generar, satisfacción en los consumidores fijando un precio bajo con calidad del producto.

\section{Estrategias competitivas.}

Alianza estratégica. Soporte Institucional por parte del gobierno. Marcas y Patentes.

\section{Elaboración de Plan de Negocios I}

El Plan de negocios en un documento escrito que define con claridad los objetivos de un negocio y describe los métodos que se emplearan para alcanzarlos.

\section{Clasificaciones del Plan de Negocios}

Plan resumido. Es el proyecto del plan con el cual se busca los fondos para el negocio, síntesis de lo que se quiere lograr. Plan completo es el plan definido, con el cual se busca captar socios. Plan Operativo: Utilizado generalmente para la ampliación de un negocio.

\section{Elaboración de Plan de Negocios II.}

Visión. - ¿Adónde quieres llegar con tu negocio?, Misión.- ¿Que harás para llegar a tu meta?, Objetivos.- Puntos concretos para cumplir con las metas, Estrategias.- Como 
cumplir los puntos concretos del plan, Plan de acción,- realizados las pautas anteriores, consiste mantener el impulso para alcanzar los objetivos y metas.

\section{Estrategias Para Competir.}

Es la manera de buscar cómo enfrentar a los negocios rivales en un determinado, entorno y mercado. Teniendo en cuenta la rivalidad dentro del sector entre los competidores existentes, amenaza de productor sustituto.

Tipos de estrategias de Competitividad: Estrategias liderazgo en costos. Estrategia en diferenciación. Estrategias e enfoque.

\section{Emprendimiento y Liderazgo.}

Son dos conceptos ligados entre si, la primera se identifica como un agente que es propenso a emprender, sea cual sea el entorno donde interactúe, sea por cuenta propia o ajena, y el segundo concepto es influir en los demás para que voluntariamente, se pueda cumplir con las metas y objetivos.

\section{Marketing}

Presentar un modelo de negocio que resulte de utilidad para la MyPE, mediante el cual va a lograr mayores ganancias, generando experiencia para futuras transacciones con clientes de diferentes partes del mundo y encontrar un espacio estable en el mercado.

\section{Explora y conquista tu mercado.}

Elaboración del Plan estratégico de Marketing. Instrucción sobre las metas que quiere alcanzar y las acciones estratégicas de la MyPE que orientan a la búsqueda de satisfacción al cliente.

Conocimiento del Consumidor. Relacionado con el comportamiento en el transcurso de compra por ser quién tiene la decisión final sobre el éxito o fracaso de un producto o servicio. Identificando los factores económicos, psicológicos y sociológicos que rigen sus estilos de vida, basados en estudios renovadores que deben tomar en cuenta la MyPE para lograr la satisfacción de los consumidores y potenciales clientes.

\section{Marketing Digital.}

Se conoce como la aplicación de las estrategias de comercialización producidas en los medios digitales. En el cual se utilizan dispositivos electrónicos, utilizando como medios, páginas web, correo electrónico, aplicaciones web, redes sociales señales de televisión y radiodifusión.

¿Cómo promocional a través del Facebook? 
Las publicaciones sobre los productos o servicios de los negocios mediante esta red social, son una manera sencilla, rápida y amplia, para dar a conocer el producto.

Pasos según la página web del Facebook, para promocionar los productos:

Paso 1: publica contenido interesante, Paso 2: haz clic en "Promocionar publicación", Paso 3: Elige tu público, Paso 4: fija el presupuesto, Paso 5: promociona tu publicación, Paso 6: Analiza los resultados.

\section{Servicio al Cliente.}

Es el conjunto de actividades que realiza la empresa para brindar el servicio o producto, con el fin de que el cliente quede satisfecho.

Funciones para una buena atención al cliente: Crear y mantener la relación con cada cliente y entenderlos, coordinar los equipos internos y externos que participan en la elaboración de la campaña, hacer presentaciones del servicio o producto, generar nuevos negocios

\section{Finanzas}

Uno de los Principales retos de la MyPE es el de enfrentar el reto de financiamiento ya se apara iniciar, ampliar o superar deficiencias económicas en el negocio, el cual lo induce a buscar fuentes de financiamiento en las diversas entidades bancarias, en este módulo se dará orientación que permita optimizar el proceso de toma de decisiones para conseguir dicho financiamiento.

\section{Se consideran los siguientes puntos:}

Determinación de la inversión. Se considera tres rubros: activos tangibles (maquinarias, equipos, terrenos, edificaciones, e instalaciones), capital de trabajo (compra de materiales e insumos, producción, ventas, y cobranza de las factura o boletas de venta), y activos intangibles (honorarios pagados para llevar estudios técnicos - económicos, pago de intereses y gastos pre-operativos, patentes, regalías entre otros).

Líneas De Financiamiento de COFIDE. Brinda financiamientos por intermedio de otros intermediarios financieros, convirtiéndose en una "Institución de segundo piso", así mismo, cuenta con programas de Servicios de información y asesoramiento a la pequeña y micro empresa.

Escenarios de la Demanda De Créditos para la MyPE. Según la Asociación de Bancos en su documento "Financiamiento Bancario para las Pequeñas y Microempresas" establece las siguientes limitaciones para que las PYME'S logren un mayor acceso al 
crédito bancario: El alto porcentaje de micro y pequeñas empresas en condiciones de informalidad, la falta de información, los trámites documentarios, la carencia de garantías.

\section{Aprendiendo el manejo de caja.}

Instrucción esencial sobre herramientas de control en el manejo de caja, el cual permitirá, evaluar si las ventas son suficientes para cubrir los gastos.

Control de caja: Registro de movimiento de dinero, separar los gastos del negocio de los gastos familiares, verificar cuánto dinero hay al inicio de la jornada diaria, programar los pagos y elimina los gastos innecesarios, control de stock, producto mayor y menor vendido, reducción de los plazos de cobro por las ventas a crédito.

\section{Desarrollo de Capacidades Emprendedoras}

\section{Emprendimiento}

Según Formichella (2004), manifiesta que "Ser emprendedor significa ser capaz de crear algo nuevo o de dar un uso diferente a algo ya existente, y de esa manera generar un impacto en su propia vida y en la de la comunidad en la que habita. A su vez, a este individuo no sólo le surgen ideas, sino que también es lo suficientemente flexible como para poder adaptarlas y posee la creatividad necesaria para transformar cada acontecimiento, sea positivo o negativo, en una oportunidad.

Así mismo, Peter (1986), expresa que: "El empresariado innovador ve el cambio como una norma saludable. No necesariamente lleva a cabo el cambio él mismo. Pero (y esto es lo que define al empresariado innovador) busca el cambio, responde a él y lo explota como una oportunidad".

\section{Capacidades emprendedoras.}

Según Román (2005), quien participó en el desarrollo del Manual para el Desarrollo de Capacidades Emprendedoras de los Adolescentes para la Fundación del Grupo romero, la define como: "La capacidad de actuar con iniciativa y perseverancia de modo de poder modificar la realidad siendo un agente de cambio, junto a los que lo rodean aportando soluciones innovadoras a organizaciones productivas y sociales desde el rol que ocupan".

Dentro de las capacidades emprendedoras que se consideran el proyecto son:

El programa de charlas empresariales tomara en el Conocimiento sobre MyPES. 
El estudiante debe tener conocimientos básicos sobre las Micro y pequeñas empresas (MyPES), la cual es la unidad económica constituida por una persona natural o jurídica, bajo cualquier forma de organización o gestión empresarial contemplada en la legislación vigente, que tiene como objeto desarrollar actividades de extracción, transformación, producción, comercialización de bienes o prestación de servicios.

Asimismo, se tomará en cuenta las capacidades empresariales de las que hace mención Román (2005). Creatividad: Es el proceso que transforma constructivamente la realidad en algo nuevo y original. En general, es "mirar" un problema o situación de una manera diferente a los demás. Este proceso de transformación constructiva empieza en la forma como se aproxima y se percibe la realidad, y culmina en la forma en cómo se modifica, logrando algo innovador y efectivo. Una condición importante es que la creatividad requiere de un pensamiento flexible que se orienta hacia la diversidad de ideas, es decir reconocer que no hay una única respuesta posible. Román (2005). Es por ello que decimos que la creatividad es una capacidad compleja y que requiere de los siguientes componentes:

Cognitivos: Entre los aspectos cognitivos de la creatividad se encuentran el pensamiento divergente y las habilidades de transformación.

Román (2005), señala la existencia de dos tipos de pensamiento, siendo estos:

Pensamiento Convergente: Se mueve buscando una respuesta determinada o convencional (lo que otros autores llaman pensamiento lógico o vertical).

Pensamiento Divergente: Se orienta en diferentes direcciones para encontrar la mejor solución. Implica un afronte flexible evaluando la situación desde diferentes ángulos, y una respuesta original, no estereotipada, que se deriva de la fluidez en el uso de los recursos personales y los que se tienen a la mano.

Características de una persona creativa. Se caracteriza por acercarse con una mirada que permite buscar y encontrar lo que otros no ven: es indagar, proponer, cambiar y atreverse.

Es una persona curiosa, inquieta, con apertura a la experiencia y a la búsqueda y la exploración. Se cuestiona y pregunta.

Es una persona ingeniosa e innovadora: Piensa e imagina algo nuevo no presentado antes, se propone alternativas novedosas, únicas, raras y de calidad. 
Generadora de cambios: Tiene una postura flexible para aceptar y proponer cambios no aceptando todo lo que le proponen.

La creatividad, así entendida, ayuda a redefinir los problemas, a no limitarnos a aceptar lo que se nos dice acerca de cómo deben hacerse las cosas y cómo hemos de pensar. Lo importante aquí es que el uso que se haga de la creatividad sea constructivo, es decir, brinde algún tipo de beneficio o satisfacción a las personas.

Iniciativa. Según Román (2005), la iniciativa es el empuje y la independencia para actuar sin necesidad que los demás te presionen o estén detrás de uno para empezar determinada actividad o brindar un comentario o idea.

La iniciativa requiere de autonomía e independencia. Implica dar el primer paso para resolver problemas u obtener logros, para arriesgarse en una acción constructiva. Es tener la actitud y disposición personal para protagonizar, promover, desarrollar ideas y emprender actividades. La persona emprendedora asumirá riesgos, pero estos serán calculados ya que el emprendimiento de una empresa no se lleva a cabo de manera aleatoria o improvisando. Para ello es importante acompañar la iniciativa con la planificación y el establecimiento claro de metas u objetivos.

\section{Características de una persona con iniciativa}

Propone, participa, actúa antes que los demás y es motivada, pues se caracteriza por una actitud proactiva ante la vida. Poseer iniciativa permite Propone y participa: no espera a que otros vengan a resolvernos los problemas. Piensa en alternativas, opina, resuelve y se muestra dispuesta para la acción.

\section{Trabajo en equipo.}

Según Román (2005), “el trabajo en equipo es el elemento fundamental para el desarrollo de las capacidades emprendedoras pues permite a las personas tener un objetivo común donde cada uno aporta con sus habilidades desde el rol que tiene". La persona emprendedora no va a trabajar sola, sabe que todo trabajo se hace en equipo, por ello, contará con otras personas que realizarán diversas labores dentro del grupo, es decir buscará formar un equipo que persiga un objetivo común. La capacidad de trabajo en equipo está relacionada con la calidad de las interacciones que establecemos con los demás. Para lograr buenos resultados se requiere de habilidades sociales y de comunicación. No basta con estar juntos porque el equipo es más que eso. Por ello, una condición importante es el establecimiento de objetivos y propósitos compartidos que 
deben ser asumidos por todos, eso es lo que permite conformar realmente un equipo. No todos hacen lo mismo: hay una complementariedad, cada uno aporta desde sus capacidades, habilidades y las responsabilidades que ha asumido. En ese sentido, además de las habilidades para interactuar con los demás, es importante la capacidad de motivar al equipo y de guiarlo.

\section{ESTRATEGIAS METODOLÓGICAS O MATERIALES Y MÉTODOS}

En la investigación se planteó se planteó una investigación cuantitativa, de tipo experimental aplicada y a la vez interpretativa, debido a que se ejecutó un Programa de charlas empresariales para el desarrollo de capacidades emprendedoras en alumnos de quinto grado de nivel secundario. La población estaba conformada por 25 estudiantes entre las edades de 16-17. La muestra estaba conformada por el total de la población. Se excluye a los estudiantes del quinto año de secundaria cuyas edades son menores a 16 años.

Las técnicas utilizadas han sido la observación directa y ficha de encuesta. Revisión del dato se examinó en forma crítica cada uno de los instrumentos utilizados con el fin de comprobar la integridad de la información, La tabulación se aplicó el programa Spps. Para la obtención de los datos.

\section{RESULTADOS Y DISCUSIÓN}

\section{RESULTADOS}

Capacidad emprendedora de los alumnos del quinto año de secundaria de la I.E. Nº381 “Ángel Custodio García Ramírez antes de la aplicación del programa de charlas empresariales.

\section{Conocimiento del tema}

Antes de la aplicación del programa de charlas empresariales; 3 estudiantes que representan el $12 \%$ indicaron que el conocimiento del tema antes de la aplicación del programa de charlas empresariales fue "Nada", mientras que 12 estudiante que representan el 48\% indicaron que fue "Poco", 5 estudiantes $(20 \%)$ indicaron que fue "Regular", 3 estudiantes que representan el 12\% indicaron que el conocimiento del tema antes de la aplicación del programa de charlas empresariales fue "Mucho" y solamente 2 estudiantes que representan el $8 \%$ indicaron que fue "Bastante".

\section{Creatividad}

Frente a la dimensión creatividad; 4 estudiantes que representan el 16\% indicaron que su creatividad antes de la aplicación del programa de charlas empresariales fue "Nada", 
mientras que 10 estudiante que representan el 40\% indicaron que fue "Poco", 4 estudiantes (16\%) indicaron que fue "Regular", 4 estudiantes que representan el 16\% indicaron que su creatividad antes de la aplicación del programa de charlas empresariales fue "Mucho" y solamente 3 estudiantes que representan el 12\% indicaron que fue "Bastante".

\section{Iniciativa}

En la presente dimensión; 2 estudiantes que representan el $8 \%$ indicaron que su iniciativa antes de la aplicación del programa de charlas empresariales fue "Nada", mientras que 6 estudiante que representan el 24\% indicaron que fue "Poco", 11 estudiantes (44\%) indicaron que fue "Regular", 3 estudiantes que representan el 12\% indicaron que su iniciativa antes de la aplicación del programa de charlas empresariales fue "Mucho" y solamente 3 estudiantes que representan el 12\% indicaron que fue "Bastante".

\section{Trabajo en equipo}

Así mismo, en su dimensión "Trabajo en equipo" antes de la aplicación del programa de charlas empresariales; 2 estudiantes que representan el $8 \%$ indicaron que el trabajo en equipo antes de la aplicación del programa de charlas empresariales fue "Nada", mientras que 12 estudiante que representan el 48\% indicaron que fue "Poco", 6 estudiantes (24\%) indicaron que fue "Regular", 3 estudiantes que representan el 12\% indicaron que el trabajo en equipo antes de la aplicación del programa de charlas empresariales fue "Mucho" y solamente 2 estudiantes que representan el $8 \%$ indicaron que fue "Bastante".

Tabla 1 Resumen de la variable desarrollo de capacidades empresariales

\begin{tabular}{lccccccc}
\hline $\begin{array}{l}\text { Desarrollo de Capacidades } \\
\text { Empresariales }\end{array}$ & \multicolumn{4}{c}{ Escala Valorativa } & \multicolumn{2}{c}{ Total } \\
& Nada & Poco & Regular & Mucho & Bastante \\
\hline Conocimiento del tema & 3 & 12 & 5 & 3 & 2 & 25 \\
Creatividad & 4 & 10 & 4 & 4 & 3 & 25 \\
Iniciativa & 2 & 6 & 11 & 3 & 3 & 25 \\
Trabajo en equipo & 2 & 12 & 6 & 3 & 2 & 25 \\
Promedio & 2.75 & 10 & 6.5 & 3.25 & 2.5 & 25 \\
Porcentaje & $11 \%$ & $40 \%$ & $26 \%$ & $13 \%$ & $10 \%$ & $100 \%$ \\
\hline Fuente: Base de datos elaborado por el autor - I.E $N^{\circ} 0381$ "Ángel Custodio García
\end{tabular}


La variable Capacidad emprendedora antes de la aplicación del programa de charlas empresariales; 3 estudiantes que representan el 11\% indicaron que su Capacidad emprendedora antes de la aplicación del programa de charlas empresariales fue "Nada", mientras que 10 estudiante que representan el 40\% indicaron que fue "Poco", 7 estudiantes (26\%) indicaron que fue "Regular", 3 estudiantes que representan el 13\% indicaron que su Capacidad emprendedora antes de la aplicación del programa de charlas empresariales fue "Mucho" y solamente 3 estudiantes que representan el 10\% indicaron que fue "Bastante".

Capacidad emprendedora de los alumnos del quinto año de secundaria de la I.E. ํㅜ 0381 “Ángel Custodio García Ramírez después de la aplicación del programa de charlas empresariales.

\section{Conocimiento del tema}

La variable Capacidad emprendedora en su dimensión "Conocimiento del tema" después de la aplicación del programa de charlas empresariales; 2 estudiantes que representan el $8 \%$ indicaron que el conocimiento del tema después de la aplicación del programa de charlas empresariales fue "Nada", mientras que 5 estudiante que representan el 20\% indicaron que fue "Poco", 5 estudiantes $(20 \%)$ indicaron que fue "Regular", 11 estudiantes que representan el 44\% indicaron que el conocimiento del tema después de la aplicación del programa de charlas empresariales fue "Mucho" y solamente 2 estudiantes que representan el $8 \%$ indicaron que fue "Bastante".

\section{Creatividad}

La dimensión Creatividad, después de la aplicación del programa de charlas empresariales; 2 estudiantes que representan el $8 \%$ indicaron que su creatividad después de la aplicación del programa de charlas empresariales fue "Nada", mientras que 2 estudiante que representan el $8 \%$ indicaron que fue "Poco", 3 estudiantes (12\%) indicaron que fue "Regular", 12 estudiantes que representan el 48\% indicaron que su creatividad después de la aplicación del programa de charlas empresariales fue "Mucho" y solamente 5 estudiantes que representan el $20 \%$ indicaron que fue "Bastante".

\section{Iniciativa}

Por su parte, la dimensión Iniciativa, después de la aplicación del programa de charlas empresariales; 2 estudiantes que representan el $8 \%$ indicaron que su iniciativa después 
de la aplicación del programa de charlas empresariales fue "Nada", mientras que 3 estudiante que representan el 12\% indicaron que fue "Poco", 6 estudiantes (24\%) indicaron que fue "Regular", 9 estudiantes que representan el 45\% indicaron que su iniciativa después de la aplicación del programa de charlas empresariales fue "Mucho" y solamente 5 estudiantes que representan el 20\% indicaron que fue "Bastante".

\section{Trabajo en equipo}

La presente dimensión, después de la aplicación del programa de charlas empresariales; 2 estudiantes que representan el $8 \%$ indicaron que el trabajo en equipo después de la aplicación del programa de charlas empresariales fue "Nada", mientras que 6 estudiante que representan el 24\% indicaron que fue "Poco", 9 estudiantes (36\%) indicaron que fue "Regular", 5 estudiantes que representan el 20\% indicaron que el trabajo en equipo después de la aplicación del programa de charlas empresariales fue "Mucho" y solamente 3 estudiantes que representan el 12\% indicaron que fue "Bastante".

Tabla 2: Capacidad emprendedora de los estudiantes después de la aplicación del programa de charlas empresariales.

\begin{tabular}{lcccccc}
\hline $\begin{array}{l}\text { Desarrollo de } \\
\text { Capacidades } \\
\text { Empresariales }\end{array}$ & Nada & Poco & Regular & Mucho & Bastante \\
\hline Conocimiento del Tema & 2 & 5 & 5 & 11 & 2 & 25 \\
Creatividad & 2 & 3 & 3 & 12 & 5 & 25 \\
Iniciativa & 2 & 3 & 6 & 9 & 5 & 25 \\
Trabajo En Equipo & 2 & 6 & 9 & 5 & 3 & 25 \\
Promedio & 2 & 4.25 & 5.75 & 9.25 & 3.75 & 25 \\
Porcentaje & $8 \%$ & $17 \%$ & $23 \%$ & $37 \%$ & $15 \%$ & $100 \%$ \\
\hline
\end{tabular}

Fuente: Base de datos elaborado por el autor - I.E $N^{\circ} 0381$ "Ángel Custodio García Ramírez": Tarapoto - 2016

El número promedio y porcentaje de estudiantes por escala valorativa de la variable Capacidad emprendedora después de la aplicación del programa de charlas empresariales; 2 estudiantes que representan el $8 \%$ indicaron que su Capacidad emprendedora después de la aplicación del programa de charlas empresariales fue "Nada", mientras que 4 estudiante que representan el 17\% indicaron que fue "Poco", 6 estudiantes (23\%) indicaron que fue "Regular", 9 estudiantes que representan el 37\% indicaron que su Capacidad emprendedora después de la aplicación del programa de 
charlas empresariales fue "Mucho" y solamente 4 estudiantes que representan el 15\% indicaron que fue "Bastante".

Influencia de la aplicación del programa de charlas empresariales en el desarrollo de las capacidades emprendedoras de los alumnos de quinto año de secundaria de la I.E. $\mathrm{N}^{\circ} 0381$ "ANGEL CUSTODIO GARCÍA RAMÍREZ" de la ciudad de Tarapoto, 2016.

\section{Prueba de hipótesis para la diferencia de medias}

Se tienen dos poblaciones y se toman muestras independientes de tamaños $\mathrm{n} 1 \mathrm{y} \mathrm{n} 2$, en este caso las muestras son de tamaño 25; se puede comparar el comportamiento de dichas poblaciones a través de los promedios.

Datos: Los resultados es la suma total de los ítems por cuestionario en ambos test.

Tabla 3: Resultados para la Prueba de Hipótesis

\begin{tabular}{|c|c|c|}
\cline { 2 - 3 } \multicolumn{1}{c|}{} & Pre test & Post test \\
\hline Promedio & 46.56 & 56.04 \\
\hline Varianza & 249.84 & 179.96 \\
\hline $\mathbf{n}$ & 25 & 25 \\
\hline $\mathbf{T}=$ & $-\mathbf{2 . 2 9}$ & \multicolumn{1}{c}{} \\
\cline { 1 - 2 } & &
\end{tabular}

Fuente: Base de datos - SPSS VER 21.

$\mathrm{T}$ probabilístico $=\mathrm{Al}$ 95\% de confianza con 48 grados de libertad $=-1.68$

$\mathrm{T}$ observado $=-2.29$ (aplicando fórmula)

Gráfico 1: Zonas de decisión Probabilística

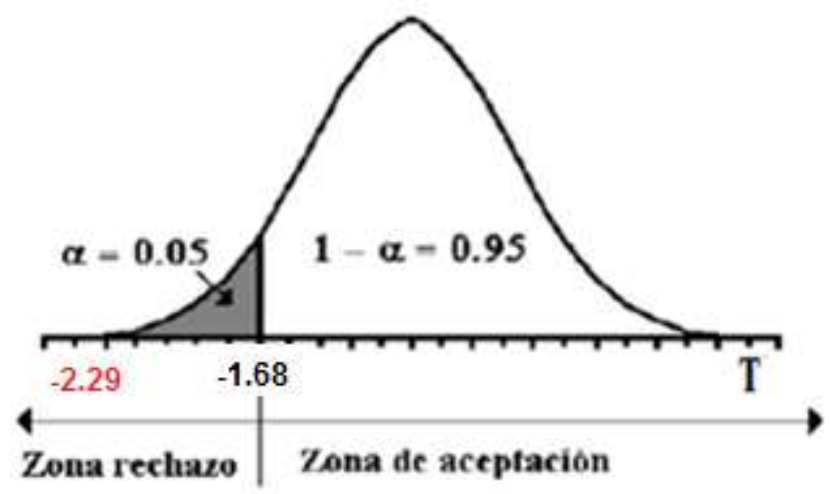

Decisión. Como nuestro T observado (-2.29) es menor que nuestro T probabilístico (1.68); se encuentra en la zona de rechazo, entonces rechazamos $\mathrm{H}_{0}$ (Hipótesis nula) y aceptamos la hipótesis alternativa, por lo tanto concluimos que: Existe efecto significativo en la aplicación del programa de charlas empresariales para el desarrollo 
de las capacidades emprendedoras de los alumnos de quinto año de secundaria de la I.E. № 0381 “ANGEL CUSTODIO GARCÍA RAMÍREZ” de la ciudad de Tarapoto, 2016 con un $95 \%$ de confianza.

\section{DISCUSIÓN}

El desarrollo de capacidades sociales y laborales, como la capacidad emprendedora de los estudiantes, supone una base fundamental para permitir que los alumnos puedan defenderse dentro de la sociedad y el mundo laboral, de esta manera en la presente investigación se pretendió evaluar la capacidad emprendedora de los alumnos del quinto año de educación secundaria de la I.E. N 0381 Ángel Custodio García Ramírez antes de la aplicación del programa de charlas empresariales, por lo que luego de haber desarrollado el cuestionario se llegó a determinar que los estudiantes presentan dificultades a la hora de demostrar sus capacidades emprendedoras ya que desconfían mucho de sí mismos, este problema se ve empeorado debido a que tanto compañeros como docentes no los apoyan y por el contrario, mencionan que su idea es poco original.

Los resultados encontrados en este trabajo difieren al de lo encontrado por Rimachi (2011), quien determina que el desarrollo de capacidades emprendedoras tiene mejores resultados si esto son desarrollados en grupos y no de forma individual, así mismo, cabe mencionar que los resultados contradicen la teoría mencionada por Formichella (2004), ya que este sugiere que el emprendimiento o las capacidades del emprendimiento, se deben al hecho de proporcionar ideas diferentes a las existentes, para lo cual deberá de ser flexible con la creatividad que posee.

Así mismo, en el presente estudio se procedió a evaluar la capacidad emprendedora de los alumnos del quinto año de secundaria de la I.E. $\mathrm{N}^{\circ} 0381$ “Ángel Custodio García Ramírez después de la aplicación del programa de charlas empresariales, y los resultados obtenidos fueron satisfactorios, ya que no solo se determinó que estas charlas mejoraron sus capacidades emprendedoras, sino también desarrollaron sus capacidades personales y sociales brindándoles mayor seguridad en sí mismos. Estos resultados guardan relación con lo encontrado por Casanovas (2009), ya que esta investigadora menciona que tras la aplicación del programa, los sujetos de muestra presentaron mejoras considerables en sus capacidades tanto personales como interpersonales, así mismo, el estudio se acoge en la teoría proporcionada por Román (2005), quien define a 
las capacidades emprendedoras como aquella capacidad de actuar con iniciativa y perseverancia de modo de poder modificar la realidad siendo un agente de cambio, junto a los que lo rodean aportando soluciones innovadoras a organizaciones productivas y sociales desde el rol que ocupan.

Por último, en la investigación lo que se hizo fue comparar los resultados obtenidos en ambos periodos, por lo que se pudo evidenciar que antes de la aplicación de las charlas empresariales, los estudiantes no tenían la capacidad de emprendimiento, sin embargo luego de la aplicación se evidenció una notable mejora. De esta manera, los resultados del estudio, guardan relación con lo encontrado por Fandiño \& Bolívar (2009), quien termina su estudio poniendo hincapié en la necesidad que tienen los estudiantes de incluir el emprendimiento como algo inherente al pensamiento de Administración de Empresas, pues es importante que se inculque en los alumnos desde un inicio con el fin de despertar la mentalidad emprendedora e innovadora; estos resultados también guardan relación con lo mencionado por Román (2005), ya que es este autor quien define a la capacidad de actuar con iniciativa y perseverancia de modo de poder modificar la realidad siendo un agente de cambio, junto a los que lo rodean aportando soluciones innovadoras a organizaciones productivas y sociales desde el rol que ocupan.

\section{CONCLUSIÓN O CONSIDERACIONES FINALES}

Las charlas empresariales dictadas a los estudiantes del quinto año del nivel secundario han desarrollado sus capacidades empresariales, es decir al realizar la comparación entre los resultados del Pre y Post - Test se ha podido identificar una significativa mejoría en las capacidades de emprendimiento concluyendo; la existencia de efecto significativo en la aplicación del programa de charlas empresariales para el desarrollo de las capacidades emprendedoras de los alumnos de quinto año de secundaria de la I.E. $\mathrm{N}^{\circ}$ 0381 “ANGEL CUSTODIO GARCÍA RAMÍREZ” de la ciudad de Tarapoto, 2016 con un $95 \%$ de confianza.

La investigación permitió identificar que los estudiantes antes de realizarse las charlas empresariales sus capacidades fueron: 3 estudiantes que representan el 11\% indicaron que su capacidad emprendedora antes de la aplicación del programa de charlas empresariales fue "Nada", mientras que 10 estudiante que representan el 40\% indicaron que fue "Poco", 7 estudiantes (26\%) indicaron que fue "Regular", 3 estudiantes que representan el 13\% indicaron que su capacidad emprendedora antes de la aplicación del 
programa de charlas empresariales fue "Mucho" y solamente 3 estudiantes que representan el 10\% indicaron que fue "Bastante".

Después de la implementación de las charlas empresariales dirigidos a los estudiantes del quinto año del nivel secundario, se pudo identificar que: 2 estudiantes que representan el $8 \%$ indicaron que su capacidad emprendedora después de la aplicación del programa de charlas empresariales fue "Nada", mientras que 4 estudiante que representan el $17 \%$ indicaron que fue "Poco", 6 estudiantes (23\%) indicaron que fue "Regular", 9 estudiantes que representan el $37 \%$ indicaron que su capacidad emprendedora después de la aplicación del programa de charlas empresariales fue "Mucho" y solamente 4 estudiantes que representan el 15\% indicaron que fue "Bastante".

\section{LISTA DE REFERENCIAS}

Ballina, F. (2007). La investigación de promoción de ventas en España. (1 ${ }^{\mathrm{a}}$ ed.). España: Edición Instituto Nacional de Tecnologías Educativas.

Casanovas, M. (2009). Desarrollo de capacidades en alumnos gitanos de educación primaria. (Tesis para obtener el grado de Doctor). Universidad Complutense de Madrid. Madrid, España. Recuperado de: http://biblioteca.ucm.es/tesis/edu/ucmt28686.pdf

Cervera, J. (2011). Propuesta didáctica basada en el uso del material educativo multimedia (Gpm2.0) para el desarrollo de las capacidades de matemáticas en alumnos del cuarto de secundaria. (Tesis para optar la licencia de educación). Universidad Católica Santo Toribio de Mogrovejo. Lambayeque, Perú. Recopilado de: http://tesis.usat.edu.pe/jspui/bitstream/123456789/92/1/TL_Cervera_Carrasco_J ocelyn.pdf

Delors, J. (1996). Educación, ciencia y cultura. ( $3^{\mathrm{a}}$ ed.). México: Ediciones UNESCO. Recopilado de: http://unesdoc.unesco.org/images/0010/001095/109590so.pdf

Fandiño, L. \& Bolívar, C. (2009). Evaluación del impacto del emprendimiento empresarial en los estudiantes y/o egresados de la carrera de administración de empresas de la Pontificia Universidad Javeriana y estudio de los factores de éxito de sus empresas creadas a partir de los talleres de grado. Universidad Javeriana Facultad de ciencias económicas y administrativas. Bogotá, Colombia. Recopilado de: http://www.javeriana.edu.co/biblos/tesis/economia/tesis32.pdf 
Formichella, M. (2004). El concepto de emprendimiento y su relación con la educación, el empleo y el desarrollo local. Instituto Nacional de Tecnología Agropecuaria. Buenos Aires, Argentina: Editorial Tres Arroyos. Recuperado de: http://municipios.unq.edu.ar/modules/mislibros/archivos/MonografiaVersionFin al.pdf

Hernández, R. (2008). Metodología de la investigación científica. (5 $5^{\mathrm{a}}$ ed.). México: Ediciones Mc Graw Hill.

Julián, J. (2009). Influencia de la aplicación de un programa formativo de profesores de educación física, sobre la motivación en el aula y el nivel de reflexión docente. Universidad de Extremadura. España. Recuperado de: http://biblioteca.unex.es/tesis/9788469274606.pdf

Moreno, M. (2009). Análisis de la capacidad empresarial de pequeños productores del Cantón de Guácimo, Costa Rica El caso de los productores inscritos al Programa de Desarrollo Comunitario de la Universidad EARTH. (tesis elaborada para obtener el título de Magister en Socio - economía Ambiental. Recopilado de: http://orton.catie.ac.cr/repdoc/A2927E/A2927E.PDF

Naciones Unidas (1968). Educación, recursos humanos y desarrollo en América Latina. Ediciones PNUD.

Página web: http://www.cofide.com.pe/COFIDE/productos.

Páginawebhttp://www.pqs.pe/actualidad/cofide-presenta-programa-de-capacitacionpara-emprendedores-gratuito-y-con-certificacion

Peter, D. (1986). La innovación y el empresario innovador. Barcelona: Editorial Edhasa.

Pinchot, G. (2003). ¿Qué pueden hacer los empresarios?. Recuperado de: www.pinchot.com/MainPages/SiteIndex.html

Rimachi, S. (2011). Propuesta curricular para desarrollar las capacidades emprendedoras en Enlácenme. Universidad Nacional de Educación Enrique Guzmán y Valle la Cantuta. Lima, Perú. Recuperado de: http://www.une.edu.pe/investigacion/TEC\%20TECNOLOGIA\%202010/TEC2010-147\%20RIMACHI\%20AYALA\%20SEGUNDINO.pdf

Román, R. (2005). Capacidad emprendedora-Cambio en el siglo XXI. (Tomo 1). Sevilla, España: Ediciones Emergencia. Recuperado de: 
http://aula.mass.pe/sites/default/files/manuales/manual_capacidades_emprended oras.pdf

Newstrom, J. (2009). Comportamiento humano en el trabajo. (13 ${ }^{\mathrm{a}}$ ed.). Monterrey, México: Editorial Mc Graw Hill. Recuperado de: https://es.scribd.com/doc/236909925/Comportamiento-Humano-en-El-Tra-JohnW-Newstrom

Dirección Regional de San Martin (2005). Módulo del proyecto educativo regional 2005 - 2021. Moyobamba: Presentado por la Dirección Regional de Educación. Recuperado de: https://es.scribd.com/doc/236909925/Comportamiento-Humanoen-El-Tra-John-W-Newstrom

Ministerio de Educación (2009). Diseño curricular nacional de educación básica regular. ( $2^{\text {a }}$ ed.). Lima, Perú: Presentado por el Ministerio de Educación. Recuperado de: http://www.minedu.gob.pe/DeInteres/xtras/den_2009.pdf

Consejo Nacional de Educación (2010). Proyecto educativo nacional 2009. Lima, Perú: Depósito Legal en la Biblioteca Nacional del Perú No - 2010-02494. Recuperado de: http://www.cne.gob.pe/images/stories/consejo\%201-42baja.pdf

Ronald, A. \& Marquardt, J. (2008). Comunicación organizacional: principios y prácticas para negocios y profesiones. (1 ${ }^{\mathrm{a}}$ ed.). México, D. F.: McGraw-Hill. http://www.mintra.gob.pe/contenidos/archivos/prodlab/legislacion/LEY 28015. pdf http://sisbib.unmsm.edu.pe/bibvirtual/publicaciones/indata/v02_n2/finanzas.htm https://www.facebook.com/business/a/online-sales/promoted-posts 